\title{
CD163 is a predictive biomarker for prognosis of classical Hodgkin's lymphoma in Saudi patients
}

\author{
HUDA AL SAYED AHMED ${ }^{1}$, WASIM FAWZI RASLAN ${ }^{2}$, \\ ABDEL HALIM SALEM DEIFALLA ${ }^{3}$ and MOHAMMAD DAHMANI FATHALLAH ${ }^{4}$ \\ ${ }^{1}$ Pathology and Laboratory Services Department, Johns Hopkins Aramco Healthcare, 1709 Dhahran; \\ ${ }^{2}$ Pathology and Laboratory Services Department, Johns Hopkins Aramco Healthcare, 10613 Dhahran, Saudi Arabia; \\ ${ }^{3}$ Department of Anatomy, College of Medicine and Medical Sciences; \\ ${ }^{4}$ Department of Higher Studies, Arabian Gulf University, 26671 Manama, Bahrain
}

Received May 10, 2018; Accepted April 5, 2019

DOI: $10.3892 / \mathrm{mco} .2019 .1850$

\begin{abstract}
Hodgkin's lymphoma (HL) is a hematological malignancy characterized by a minority of neoplastic cells outnumbered by tumor-associated macrophages (TAMs). The overexpression of the CD163 antigen by TAMs is considered to be a significant predictive biomarker for risk stratification. This is likely caused by a genetic single-nucleotide polymorphism (SNP) at the gene promoter. The aim of the present retrospective case-control study was to establish a gene expression profile of a specific biomarker for classical HL (CHL) in order to predict the outcome and survival of CHL patients in Saudi Arabia. The protein expression of CD163 on TAMs was studied using immunohistochemistry (IHC). A prognosis index was calculated for the CD163 protein to assess the risk stratification of CHL. Genotyping of selected SNPs of this antigen was performed for $100 \mathrm{CHL}$ cases and controls. The analysis revealed that the CD163 protein expression level was significantly correlated with disease relapse (DR) and overall survival (OS). In addition, the CD163 index threshold (15.0) was found to be significantly correlated with the relapse rate. Among the studied CD163 SNPs, rs75608120 exhibited a significant correlation with the DR rate of CHL patients, but not with OS. The findings of the present study confirmed that CD163 is a specific marker for TAMs, and its overexpression by TAMs is significantly associated with relapse and reduced survival post-therapy. In addition, a new methodology of indexing CD163 protein expression for HL risk stratification was proposed. Thus, the present study identified a specific
\end{abstract}

Correspondence to: Dr Huda Al Sayed Ahmed, Pathology and Laboratory Services Department, Johns Hopkins Aramco Healthcare, 6th Street, Aramco Gharb Al Dhahran, Az Zahran 34465, 1709 Dhahran, Saudi Arabia

E-mail: huda.alsayedahmed@jhah.com

Key words: classical Hodgkin's lymphoma, tumor-associated macrophages, immunohistochemistry, CD163, predictive biomarkers predictive molecular and antigenic biomarker for $\mathrm{CHL}$ prognosis.

\section{Introduction}

Hodgkin's lymphoma (HL) is a clonal B-lymphocyte neoplasm commonly affecting younger patients. HL has characteristic pathological stages, highlighting the paramount importance of a timely and effective clinical management. Epidemiological studies on HL from Saudi Arabia are scarce (1). Recent cancer researchers have yet to identify tumor-specific biomarkers with inter-individual variations (i.e., polymorphisms). It has been hypothesized that genetic signatures may enable genetic prediction of cancer prognosis. Obviously, this successful prediction will determine the diagnostic and prognostic value of the association between the biomarker and the clinical outcome (2). HL is the ninth most common malignancy in the Gulf Cooperation Council (GCC) States, and its incidence has continuously increased over the past decade among GCC nationals (3). The 2013 Saudi Cancer Registry report revealed that the median age of HL at diagnosis was 25 years (4). In most Western countries, HL has two peaks of incidence, at the age range of 10-35 years old and at a later age $>60$ years old. However, in the Middle East and Asia, HL is more common during childhood (5). Based on a study by Al-Diab et al (6) on a Saudi and Middle Eastern population, it is hypothesized that the rapid improvement in the living standards and healthcare systems over the past two decades has affected the clinical behavior of HL.

The diagnosis of HL is confirmed by the presence of Hodgkin and Reed-Sternberg cells (HRS) $(7,8)$. The Revised European-American Lymphoma (REAL) classification, which was later adopted by the World Health Organization (WHO) scheme, concluded that HL comprises two well-defined entities: Nodular lymphocyte-predominant (NLP) HL and the more common variant, classical HL (CHL), which was sub-classified into four subtypes: Nodular sclerosis (NS), mixed-cellularity (MC), lymphocyte-depleted (LD), and lymphocyte-rich (LR) (7,9). The diagnosis of HL should be followed by staging, which is achieved using the Ann Arbor staging scheme or its Cotswold modification; the latter classifies 
HL as low-grade (stages I and II) and high-grade (stages III and IV) (10). To date, the most widely accepted risk stratification scoring system for high-grade HL is the International Prognostic Score (IPS), with a higher score predicting a worse outcome (11-13). Another prognostic factor for early-stage HL is the erythrocyte sedimentation rate (ESR); an increased ESR is predictive of early relapse and poor prognosis (14).

The diagnosis of HL relies on morphological examination and immunophenotyping of the HRS cells. This may be achieved by immunohistochemical (IHC) staining of formalin-fixed paraffin-embedded (FFPE) sections, or the multi-parametric flow cytometry technique for fresh tissue specimens. These reliable assays display the same accuracy, and can directly detect HRS lymphoma cells and evaluate the background cells as an adjunct diagnostic test (15). Flow cytometry enables sensitive detection of antigens for which antibodies may not be available in IHC staining. In addition to the ease in defining a distinct cell population based on their size, granularity and presence of specific tumor cell markers, it excludes dead cells. Similarly, the FFPE tissue specimens are not suitable for flow cytometric analysis, as it requires viable, unfixed cells $(8,16)$.

In most HL cases, HRS cells constitute the minority of the cellular milieu, while the vast majority of the tumor microenvironment (TME) cells are immune non-neoplastic cellular components $(9,17)$. There are four TME patterns observed that form the basis of the different CHL subtypes (17). Furthermore, TME cells are outnumbered by tumor-associated macrophages (TAMs), which participate in the recruitment of more macrophages and other immune cells (18). TAMs promote peritumoral inflammation and avoid immune destruction, as they exhibit TAM-specific tumor-sustaining properties (19). Macrophages were found to support tumor growth and suppress the immune response that targets tumor cells (20). Increased numbers of TAMs have been reported to confer a poor prognosis, inferior outcomes and shortened survival in HL (6). Ree and Kadin reported that the presence of large numbers of TAMs is correlated with a high incidence of clinical-laboratory relapse within 2 years of therapy (21). Increased numbers of TAMs in the TME should be taken into consideration to improve prognostic prediction standards and to plan appropriate therapeutic strategies (22). However, although advances in therapy may achieve a disease-free survival, this is accompanied by increased risk of secondary cancers, most commonly in HL patients with a family history of cancer (23). The currently available therapeutic protocol is unsuccessful in $20-30 \%$ of HL cases, as the current predictive systems cannot accurately identify these high-risk patients $(24,25)$. Therefore, minimizing the adverse effect of TME cells on treatment success and reducing tumor progression may be achieved via therapeutic inhibition of TAMs. Therapeutic targeting of these chemo-protective TAMs may improve the efficiency of standard treatments $(17,19,26,27)$.

The gene signature of HL is routinely validated in FFPE tissues using IHC. CD163 antigen expression by TAMs was evaluated by computer image analysis or quantified manually by pathologists. Scoring was performed by estimating the relative percentage of CD163-positive cells in relation to overall cellularity (18). The overexpression of the CD163 antigen is an important pre-treatment measure and may be used to identify patients with an unfavorable prognosis or at high risk for relapse. On the other hand, patients with a favorable prognosis should not be over-treated (28). This gene signature of TAMs was validated in several cohort studies, which have demonstrated that increased CD163 expression on IHC was directly associated with treatment failure and a poor event-free survival. The adverse prognostic significance of CD163 has been confirmed to be a predictive biomarker for HL patients $(18,20,24,29)$. Yoon et al (30) concluded that a high CD163 immunostaining intensity is particularly associated with a decreased rate of complete remission (CR) and is considered as a specific reliable prognostic indicator in HL. By contrast, Azambuja et al (31), did not observe such an association in their study population. The CD163 gene is a scavenger receptor cysteine-rich (SRCR) protein-coding gene; it is a restricted monocyte and tissue macrophage marker, as demonstrated by IHC $(32,33)$. CD163 is considered to be a better TAM marker compared with CD68 in HRS cell-rich areas, with less non-specific staining of the background (34). A single-nucleotide polymorphism (SNP) in the CD163 gene may modify the CD163 protein phenotype, particularly if these SNPs are located within the regulatory region that can alter the protein expression and, thus, the functional properties of the CD163 protein (35).

\section{Materials and methods}

Sample collection. CHL comprises the majority of $\mathrm{HL}$ cases. A review of our records (between 1990 and 2015) at John Hopkins Aramco Healthcare Center (JHAH) in Saudi Arabia revealed that CHLs represented $>85 \%$ of HL cases. Although HL may be curable, a marked percentage of HL cases (20-30\%) fail to respond to therapy. Early identification of those cases has become an important objective for clinical research. The aim of this retrospective case-control study was to investigate the significance of the CD163 antigen expression and the presence of the related SNPs on the prognosis of CHL in Saudi patients. A total of 100 formalin-fixed paraffin-embedded (FFPE) tissue samples of patients previously diagnosed with CHL were collected. Patients were selected from all histological CHL subclasses and stages. A total of 20 FFPE normal lymphoid samples (mainly tonsils) that had been collected within the same period from subjects with no known personal or family history of cancer were used as negative controls for the IHC staining evaluation. In addition, 100 EDTA peripheral blood samples were collected from healthy blood donors to be used as a study control group for the molecular genotyping assay. The study protocol received ethical approval from the Institutional Review Board at John Hopkins Aramco Healthcare Center.

IHC staining and evaluation. All the FFPE tissue blocks were re-cut into two representative sections that were mounted on slides and designated as CD30 and CD163. In addition, a normal lymph node tissue section was included as a quality control in each slide. All tissues were processed in the fully automated staining instrument Ventana Benchmark Ultra (Ventana Medical Systems, Inc., Tucson, AZ, USA) for antigen retrieval and immune staining. IHC included monoclonal antibody CD30 (clone BerH2, cat. no. 790-2926, 
Roche Diagnostics, Basel, Switzerland) and CD163 (clone 10D6, product code: NCL-L-CD163, Leica Biosystems Ltd., Newcastle, UK; dilution 1:20), using a multimeric UltraView Universal DAB detection kit and Ventana iView ${ }^{\mathrm{TM}}$ DAB Detection kit (Ventana Medical Systems, Inc.).

IHC staining was evaluated by a pathologist to select the areas that contained CD30-positive HRS cells; these were analyzed for CD163 expression on the surrounding TAMs. Sections lacking CD30-positive cells, areas with fibrosis, blood vessels, reactive lymph nodes, necrosis and artifacts were excluded from the analysis field to minimize areas of non-specific background staining. The tissue sections were examined manually using a Nikon ECLIPSE E600 microscope (Nikon Corporation, Tokyo, Japan) under x40 lenses, equivalent to 1 high-power field of $0.5 \mathrm{~mm}$ in diameter and an area of $0.196 \mathrm{~mm}^{2}$. The number of CD30-positive cells in at least 10 fields was counted and a mean was calculated. For CD163-stained slides, the number of CD163-positive monocytes/macrophages surrounding a central cluster of CD30-positive cells was counted in 10 fields, then the mean was also calculated. We herein intended to introduce a new methodology for calculating a 'prognosis index' of CD163, which was calculated by dividing the mean number of CD163-positive TAMs by the mean number of CD30-positive cells. Although CD30 IHC may stain activated B lymphocytes in addition to HRS cells, it is used as a baseline to differentiate reactive $\mathrm{B}$ lymphocytes from malignant HRS cells. CD30-positive reactive B lymphocytes were counted in the normal lymph node tissues (i.e., negative controls), selecting the inter-follicular rather than the intrafollicular areas or sinusoidal spaces, and selecting the scattered non-clustered cells in that area. CD30-positive cells were used at denominator for the calculated CD163 index to eliminate errors and yield a more specific absolute number reflecting a more realistic index value. The equation used was as follows:

CD163 prognosis index=Mean number of CD163-positive TAMs/mean number of CD30-positive cells.

Genotyping by polymerase chain reaction (PCR) analysis. All FFPE sections of the CHL and control groups were assayed for molecular PCR genotyping. Two normal tonsillar fresh tissue specimens were also obtained to serve as PCR negative controls. In addition, two fresh lymph node tissue samples from newly diagnosed CHL patients served as PCR positive controls. Total genomic DNA was extracted from patients' FFPE and control group tissues, in addition to EDTA peripheral blood samples of the study control group, using QIAamp DNA FFPE Tissue (250) kit (cat. no. 56404, Qiagen Inc., USA) and Qiagen Mini Spin column technique, according to the manufacturer's instructions and specifications.

Genotyping assay was performed for seven CD163 gene SNPs, including four randomly selected SNPs at the promoter region (rs61729, rs11054197, rs11054195 and rs75608120), in addition to three different randomly selected SNPs at the protein-coding region (rs200642325, rs61729510 and rs150018775) in both control and CHL patient samples. PCR was performed using 96-well plates and TaqMan ${ }^{\circledR}$ GTXpress ${ }^{\mathrm{TM}}$, Genotyping Assays, Human, SM Kit (cat. no. 4351379; Applied Biosystems; Thermo Fisher Scientific, Inc., Waltham,
MA, USA). The PCR reaction was performed with an Applied Biosystems 7500 Real Time PCR system following the standard manufacturer's protocols and the following conditions: Enzyme activation at $95^{\circ} \mathrm{C}$ for $20 \mathrm{sec}$, then total of 40 cycles of both denaturation at $95^{\circ} \mathrm{C}$ for $15 \mathrm{sec}$ and annealing/extension at $60^{\circ} \mathrm{C}$ for $60 \mathrm{sec}$.

Statistical analysis. All statistical analyses were performed using SPSS software, version 22.0 (IBM Corp., Armonk, NY, USA). The results were considered statistically significant at $\mathrm{P} \leq 0.05$. CD163 IHC optimum threshold was determined using SPSS software based on the maximal $\chi^{2}$ value of the log-rank test for disease relapse (DR), classified as low- and high-risk. DR was defined as the time between treatment initiation and disease progression or relapse. Overall survival (OS) was defined as the time between treatment initiation and death from any cause. The t-test was used for the association of the CD163 indices (high and low) with DR and OS. Fisher's exact probability (Chi-squared) test was used to investigate any association of all the studied SNPs with DR and OS in CHL patients. One-way analysis of variance was applied to investigate the association of the CD163 indices with different SNPs, and also for different HL stages and CHL subtypes. The correlation of the CD163 prognosis index with ESR for low-stage CHL, and with IPS score for high-stage CHL, were analyzed by t-tests.

Survival curves were constructed using the Kaplan-Meier method and assessed for statistical significance using the log-rank test. Genotype and allele frequencies for all used SNPs were estimated using the online version of SHEsis Software (http://analysis.bio-X.cn/myAnalysis.php). Fisher's exact probability (Chi-squared) test was used to evaluate any associations of all the studied SNPs with DR and OS in the CHL patients included in this study.

\section{Results}

Demographic characteristics of the studied Saudi CHL patients. Our data demonstrated that $44 \%$ of the patients were female and $56 \%$ were male, with a male: Female ratio of 1.3:1.0. The CHL patients' age at diagnosis ranged from 3 to 80 years, with a median age of 42 years. The majority $(78 \%)$ of the patients were aged $<45$ years (Table I). The aggregated data also revealed that the CHL entity constituted $85.2 \%$ of all newly diagnosed HL cases at JHAH, whereas NLP accounted for $14.8 \%$ of all cases over the past 26 years (Fig. 1). Moreover, $\sim 81 \%$ of the cases diagnosed at JHAH exhibited a higher prevalence of the NS subtype of CHL compared with the other subtypes (Table I; Fig. 2). In addition, data analysis demonstrated that stage II and III CHLs were the most common among the studied Saudi patients (41.3 and $33.7 \%$, respectively; Table I).

CD163 IHC staining. The microscopic examination of IHC staining for CD163 revealed that this protein is expressed on TAMs as surface/membranous and cytoplasmic/lysosomal staining in cases with a higher number of TAMs around HL cells; however, it was mainly expressed as cytoplasmic/lysosomal staining in cases with a lower number of TAMs surrounding HL cells. Only CD163-positive TAMs (Fig. 3B) surrounding 
Table I. Demographic characteristics of CHL cases in the Saudi population at the John Hopkins Aramco HealthCare Center during the period between 1990 and 2015.

\begin{tabular}{|c|c|c|}
\hline Demographic characteristics & Total no. (n) & $\%$ \\
\hline Age (years) & 100 & \\
\hline$<45$ & 78 & 78.0 \\
\hline$>45$ & 27 & 27.0 \\
\hline Sex & 100 & \\
\hline Male & 56 & 56.0 \\
\hline Female & 44 & 44.0 \\
\hline CHL stage & 92 & \\
\hline I & 8 & 8.7 \\
\hline II & 38 & 41.3 \\
\hline III & 31 & 33.7 \\
\hline IV & 15 & 16.3 \\
\hline CHL subtype & 100 & \\
\hline NS & 81 & 81.0 \\
\hline MC & 13 & 13.0 \\
\hline LR & 5 & 5.0 \\
\hline $\mathrm{LD}$ & 1 & 1.0 \\
\hline Treatment received (response) & 100 & \\
\hline Curative & 98 & 98.0 \\
\hline Palliative & 2 & 2.0 \\
\hline Relapse & 100 & \\
\hline No relapse & 84 & 84.0 \\
\hline Relapsed & 16 & 16.0 \\
\hline Overall survival & 100 & \\
\hline Did not survive & 11 & 11.0 \\
\hline Survived & 89 & 89.0 \\
\hline
\end{tabular}

CHL, classical Hodgkin's lymphoma; NS, nodular sclerosis; MC, mixed-cellularity; LD, lymphocyte-depleted; LR, lymphocyte-rich.

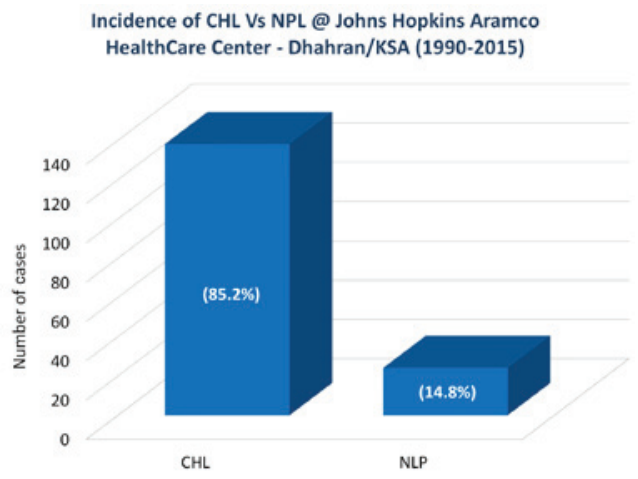

Figure 1. Prevalence of CHL vs. NLP at the John Hopkins Aramco HealthCare (JHAH) Center-KSA between 1990 and 2015. Data from JHAH Center-KSA revealed a higher number of CHL cases compared with the NLP type of HL in Saudi patients. CHL, classical Hodgkin's lymphoma; NLP, nodular lymphocyte-predominant.

a central cluster of CD30-positive cells (Fig. 3A) were taken into consideration to calculate the CD163 index in this study.

\section{Incidence of $\mathrm{HL}$ at Johns Hopkins Aramco HealthCare Center@ Dhahran-KSA (1990-2015)}

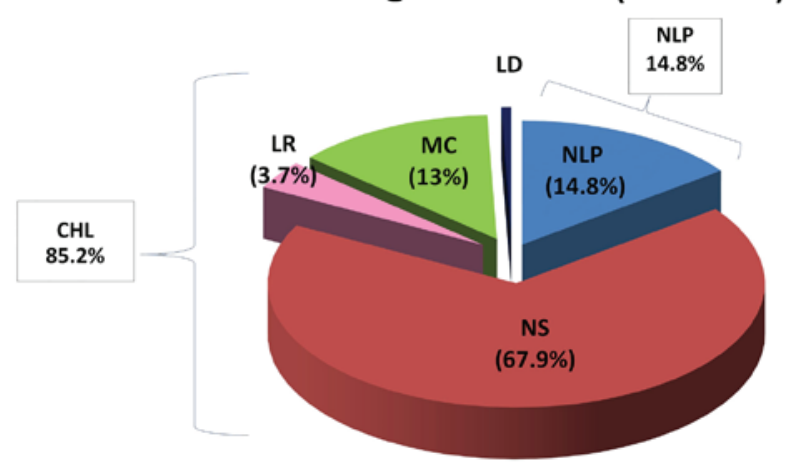

Figure 2. Prevalence of different CHL subtypes at the John Hopkins Aramco HealthCare (JHAH) Center-KSA between 1990 and 2015. Data from JHAH Center-KSA revealed a highest percentage of the NS-CHL subtype in comparison with the other subtypes in Saudi patients. CHL, classical Hodgkin's lymphoma; NLP, nodular lymphocyte-predominant; NS, nodular sclerosis; LR, lymphocyte-rich; MC, mixed-cellularity; LD, lymphocyte-depleted.

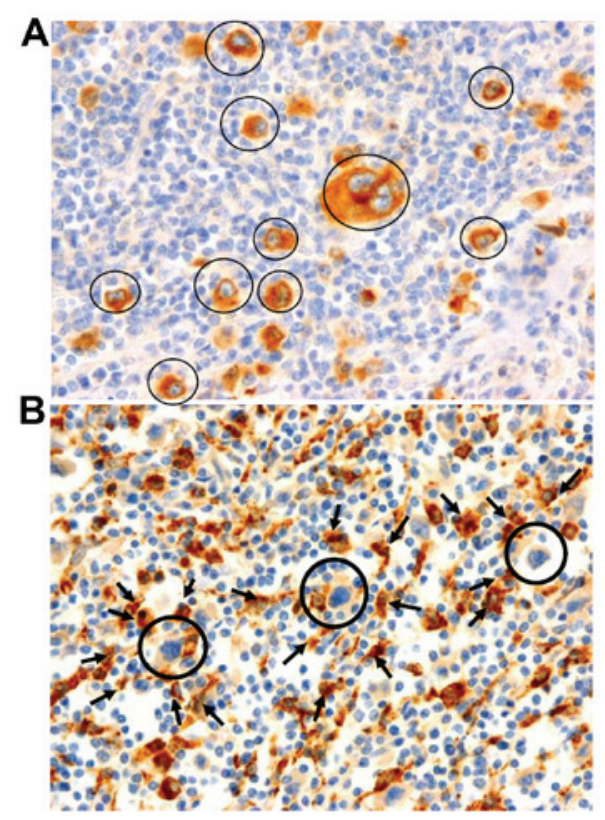

Figure 3. Immunohistochemical staining results. (A) CD30-positive HRS cells (circles). (B) CD163-positive monocytes/macrophages (arrows) surrounding the HRS cell cluster at a magnification of $x 40$. HRS, Hodgkin and Reed-Sternberg.

The CD163 prognosis index was calculated by dividing the number of CD163-positive macrophages by the total number of CD30-positive HRS cells in CHL patients, and by the number of CD30-positive reactive B-lymphocytes in the control group. When comparing the index mean of CD163 expressed on TAMs in CHL patients against that of the control group using Student's t-test, a statistically significant difference was observed $(\mathrm{P}<0.001$; Table II).

Determining the threshold of the CD163 prognosis index. The CD163 prognosis index threshold was determined using t-test to compare the CD163 IHC mean of the relapsed cases vs. that of the non-relapsed patients at several cut-off values $(5,7,10,15,20$ and 25$)$ of the CD163 index. A threshold value 
Table II. Comparison of the CD163 index between CHL patients and control subjects.

\begin{tabular}{lccc}
\hline Indices & CHL patients $(\mathrm{n}=100)$ & Controls $(\mathrm{n}=20)$ & $\mathrm{t}$-test P-value \\
\hline $\mathrm{CD} 163$ (mean) & 13.5 & 2.8 & $<0.001$
\end{tabular}

CHL, classical Hodgkin's lymphoma.

Table III. CHL risk stratification groups at the cut-off value of the CD163 index in comparison with controls.

\begin{tabular}{|c|c|c|c|c|c|}
\hline \multirow[b]{2}{*}{ CD163 IHC index (at cut-off) } & \multicolumn{2}{|c|}{ CHL patients } & \multicolumn{2}{|c|}{ Controls } & \multirow[b]{2}{*}{ P-value } \\
\hline & $\mathrm{n}$ & $\%$ & $\mathrm{n}$ & $\%$ & \\
\hline Low-risk CHL $(\leq 15)$ & 69 & 69.0 & 20 & 100.0 & $<0.001$ \\
\hline High-risk CHL (>15) & 31 & 31.0 & 0 & 0 & \\
\hline Total & 100 & 100.0 & 20 & 100.0 & \\
\hline
\end{tabular}

CHL, classical Hodgkin's lymphoma; IHC, immunohistochemistry.

of 15 reflected significant differences between the relapsed and non-relapsed patient groups, with a P-value of 0.008 . Accordingly, CHL patients were divided into two stratification groups: Low-risk (lower CD163 index, $\leq 15.0$ ) and high-risk (higher CD163 index, >15.0). In this study, 69/100 (69\%) of CHL patients had a low CD163 index and 31/100 (31\%) had a high CD163 index (Table III).

Association of CD163 IHC prognostic index with DR and $O S$ in $C H L$. The analysis revealed that there was a statistically significant direct association of the CD163 index with DR and OS, with a Pearson's Chi-squared P-value of $<0.001$. Additionally, the CD163 index at the determined threshold level (15.0) was directly associated with DR $(\mathrm{P}=0.022$, Fisher's exact test $=0.034)$; however, it was not significantly associated with $\mathrm{OS}$ rate $(\mathrm{P}=0.173$, Fisher's exact test=0.189).

Genotyping analysis of CD163 gene SNPs. Upon CD163 gene SNPs analysis, genotype and allele frequencies for all seven studied SNPs were calculated using the SHEsis Software. The analysis demonstrated that all CD163 gene SNPs were at Hardy-Weinberg equilibrium (HWE) among controls (Fisher's P-value $>0.05$ ), which indicated that our population was homogeneous, excluding rs75608120 SNP, which is located at the promoter region of the CD163 gene and was not in HWE among controls (Fisher's P-value $<0.001$ ), indicating that our population was not homogeneous for this SNP (Table IV). The theory behind HWE states that in a given population, allele frequency as well as genotype frequency of any gene will persist among consecutive generations, unless evolutionary influences interfere.

Association of CD163 gene SNPs with DR and OS of CHL. Chi-square analysis of allele genotype of the studied CD163 gene SNPs revealed that there is no statistically significant association with DR or OS in CHL, except for one CD163
SNP (rs75608120), which is located in the promoter region, and exhibited a statistically significant association with DR $(\mathrm{P}=0.032)$, but not with OS (Table V).

Testing the study hypothesis. In order to determine whether CD163 protein overexpression is stimulated by the presence of any SNP at the gene promoter region, or even at the protein-coding region, the calculated CD163 prognostic index was evaluated for its association with its SNPs using analysis of variance (ANOVA). The analysis revealed that none of the studied SNPs were associated with the CD163 IHC protein expression $(\mathrm{P}=0.690)$. Thus, the null hypothesis was rejected.

Association of CD163 IHC prognostic index with ESR for low-stage CHL. The CD163 IHC-determined index was assessed via Student's t-test for association with ESR, as this predictive biomarker is already used to assess the CHL risk for low-stage HL (I and II). The analysis demonstrated a direct significant association between the ESR and the CD163 index $(\mathrm{P}<0.001)$ : The CD163 index was higher in CHL cases with an ESR of $>20 \mathrm{~mm} / \mathrm{h}$ (Table VI).

Association of the CD163 IHC prognostic index with IPS score for high-stage CHL. The statistical t-test was used to assess the association of the IHC CD163 index with IPS, as it is the accepted tool used for predicting the HL risk for high-stage CHL (III and IV). The analysis confirmed a significant association between IPS and the CD163 index $(\mathrm{P}<0.001)$ : The CD163 index was higher in CHL cases with an IPS of $>3$ (Table VI).

CHL survival curve based on the established CD163 prognostic index threshold. The total number of relapsed and deceased CHL patients in our study was 27/100 (27\%). A total of 16 CHL patients (16\%) constituted the known relapsed CHL cases within a period ranging from 1 month to 12 years, with a median of 3.2 years. A total of 11/100 CHL patients (11\%) 


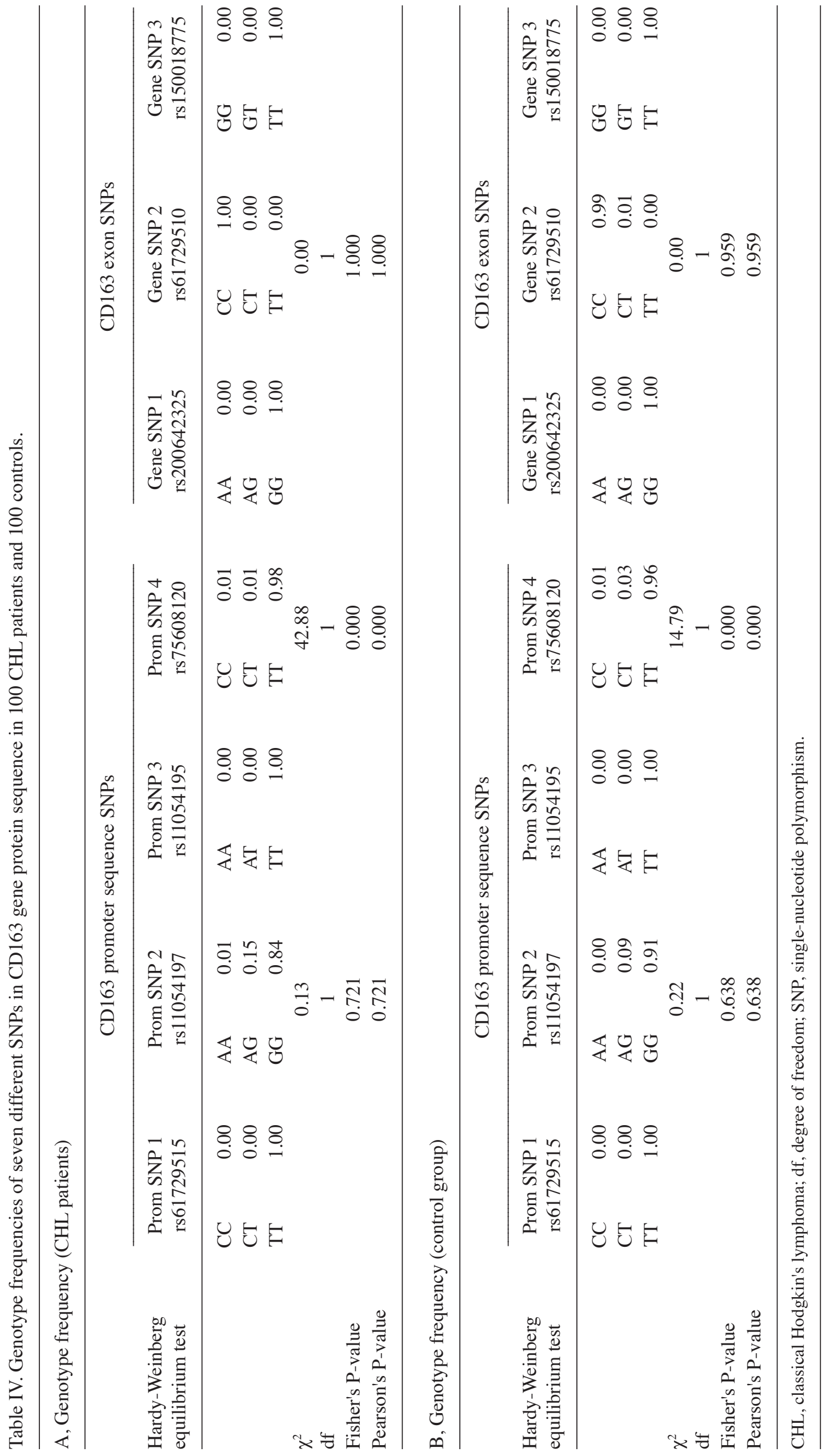


Table V. Significance of the correlation between CD163 SNPs with the DR and OS rates of the CHL cases among Saudi patients at the John Hopkins Aramco HealthCare Center during the period between 1990 and 2015.

\begin{tabular}{|c|c|c|c|c|c|c|}
\hline \multirow[b]{2}{*}{ CD163 promoter region SNPs } & \multicolumn{2}{|c|}{ CHL patients } & \multicolumn{2}{|c|}{ Control group } & \multirow{2}{*}{$\begin{array}{c}\text { DR P-value } \\
\mathrm{NC}^{\mathrm{a}}\end{array}$} & \multirow{2}{*}{$\begin{array}{c}\text { OS P-value } \\
\mathrm{NC}^{\mathrm{a}}\end{array}$} \\
\hline & $\mathrm{n}=100$ & $\%$ & $\mathrm{n}=100$ & $\%$ & & \\
\hline \multicolumn{7}{|l|}{ SNP1 (rs61729515) } \\
\hline Homozygous for wild-type allele & 0 & 0 & 0.0 & 0 & & \\
\hline Heterozygous & 0 & 0 & 0.0 & 0 & & \\
\hline Homozygous for mutant allele & 100 & 100 & 100.0 & 100 & & \\
\hline SNP2 (rs11054197) & 100 & & 100 & & 0.203 & 0.573 \\
\hline Homozygous for wild-type allele & 1 & 1 & 0.0 & 0 & & \\
\hline Heterozygous & 15 & 15 & 9.0 & 9 & & \\
\hline Homozygous for mutant allele & 84 & 84 & 91.0 & 91 & & \\
\hline SNP3 (rs11054195) & 100 & & 100 & & 0.637 & 0.637 \\
\hline Homozygous for wild allele & 0 & 0 & 0.0 & 0 & & \\
\hline Heterozygous & 0 & 0 & 0.0 & 0 & & \\
\hline Homozygous for mutant allele & 100 & 100 & 100.0 & 100 & & \\
\hline SNP4 (rs75608120) & 100 & & 100 & & 0.032 & 0.09 \\
\hline Homozygous for wild allele & 1 & 1 & 1.0 & 1 & & \\
\hline Heterozygous & 1 & 1 & 3.0 & 3 & & \\
\hline Homozygous for mutant allele & 98 & 98 & 96.0 & 96 & & \\
\hline
\end{tabular}

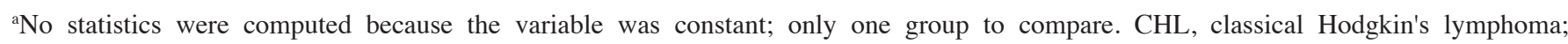
SNP, single-nucleotide polymorphism; DR, disease relapse; OS, overall survival.

Table VI. Association of ESR and IPS score with the CD163 index in HL patients.

\begin{tabular}{lrcc}
\hline Variables & $\mathrm{n}$ & $\%$ & P-value (t-test) \\
\hline ESR (stages I and II), mm/h & 46 & & $<0.001$ \\
$<20$ & 20 & 43.3 & \\
$>20$ & 26 & 56.6 & \\
IPS (stages III and IV) & 46 & & $<0.001$ \\
$<3$ & 18 & 39.1 & \\
$>3$ & 7 & 15.2 & \\
\hline
\end{tabular}

IPS was calculated based on HL stage, age, sex, white blood cell count, lymphocyte count, hemoglobin level and serum albumin. It is commonly used for advanced-stage (III and IV) HL and it was calculated for 25 of the 46 cases, while in the rest of cases the albumin level was missing. ESR, erythrocyte sedimentation rate; IPS, international prognostic score; HL, Hodgkin's lymphoma.

succumbed to the disease within the same time period. A total of $11 \mathrm{CHL}$ known cases died from the disease, the majority (9 out of 11) of them succumbed within the first 5 years, while the minority ( 2 out of 11 ) survived for $>5$ years. The median OS was $\sim 3.5$ years in our patient population (Table VII).

A survival curve was constructed for the CD163 index using the Kaplan-Meier method, which calculates the cumulative survival rate. Accordingly, the CD163 index was statistically significant in high-risk CHL patients (i.e., CD163 index
$>15.0$ ) in comparison with the low-risk patients (i.e., CD163 index $\leq 15.0$ ). Subsequently, there was an observable decline in the survival of high-risk CHL patients (Fig. 4). Similarly, the hazard function analysis demonstrated a higher cumulative hazard in the high-risk CHL group (Fig. 5). The survival and the hazard function were assessed using the log-rank test, which revealed a significant association between the CD163 index and survival rate, with a P-value of 0.039.

\section{Discussion}

In the present study, we first attempted to standardize the evaluation of CD163 expression on TAMs using IHC, and to determine a threshold level for CHL patients to help stratify disease prognosis. To investigate the effect of CD163 protein expression on CHL prognosis, the association of CD163 IHC with DR and OS was evaluated and found to be statistically significant $(\mathrm{P}<0.001)$. This finding confirms that an increased number of TAMs overexpressing CD163 is significantly associated with reduced progression-free and overall survival after standard chemotherapy (with or without radiotherapy). Therefore, this antigen may be considered as a prognostic biomarker for CHL in Saudi patients.

We attempted to determine the threshold of IHC staining level of CD163 in order to stratify the CHL cases into lowand high-risk groups. Based on the maximal $\chi^{2}$ value of the log-rank test with regard to relapse and survival rate (i.e., DR and OS), the CD163 index threshold was determined as 15.0, with a Pearson's Chi-squared P-value of 0.008 and a Fisher's exact test $\mathrm{P}$-value of 0.014 . This threshold value for CD163 
Table VII. Summary of DR and OS in the studied CHL patients at the John Hopkins Aramco HealthCare Center.

\begin{tabular}{|c|c|c|c|c|c|c|}
\hline & \multicolumn{4}{|c|}{ DR } & \multicolumn{2}{|c|}{ OS } \\
\hline & 0-6 months & 6 months -5 years & 5-10 years & $>10$ years & $<5$ years & $>5$ years \\
\hline No. of CHL patients & $3 / 100$ & $10 / 100$ & $2 / 100$ & $1 / 100$ & $9 / 100$ & $2 / 100$ \\
\hline
\end{tabular}

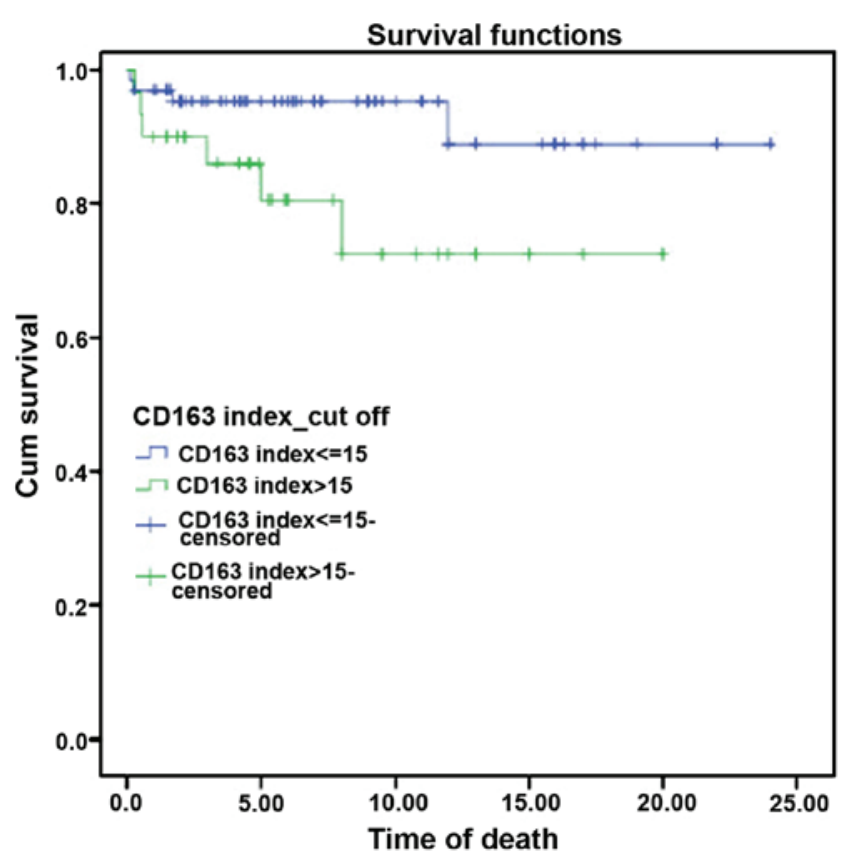

Figure 4. Estimated survival curve based on the CD163 index (time is calculated in years).

was significantly correlated with the number of relapsed CHL patients $(\mathrm{P}=0.022)$. Moreover, our study demonstrated that CD163 protein expression was significantly correlated with ESR in low-stage (I and II) and with IPS in high-stage (III and IV) CHL, with a P-value of $<0.001$. This finding supports the use of CD163 expression levels in addition to ESR and IPS as biomarkers for predicting the prognosis of CHL.

To complete the study, we performed genetic typing of 7 SNPs in the 5' region of the CD163 locus in 100 CHL patients and an equivalent number (i.e., $\mathrm{n}=100$ ) of healthy controls. The 7 polymorphisms were selected on the basis that they may guide, drive or affect the protein expression level of CD163. To the best of our knowledge, no previous study has investigated CD163 gene polymorphisms, or their correlation with the level of protein expression or CHL prognosis. We herein attempted to correlate the presence of different CD163 gene SNPs with CHL prognosis by calculating the statistical significance of these SNP genotypes with the DR and OS of CHL. Using the Chi-squared test, we found that all the studied CD163 SNPs were insignificantly correlated with DR and OS in CHL patients, except for rs75608120 (a polymorphism located in the promoter region). This SNP exhibited a significant correlation

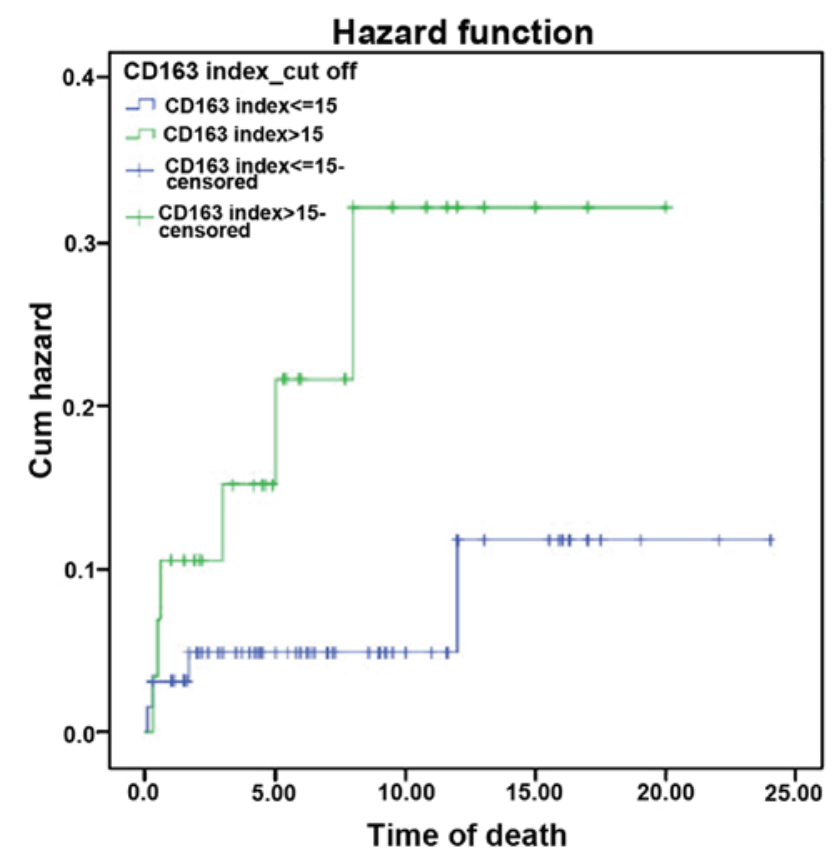

Figure 5. Estimated hazard curve based on the CD163 index (time is calculated in years).

with the DR of CHL $(\mathrm{P}=0.032)$, but not with $\mathrm{OS}(\mathrm{P}=0.090)$. One of the main goals of the present study was to determine whether CD163 protein overexpression is guided or stimulated by the presence of any one SNP in CHL patients compared with healthy controls. Upon multivariate ANOVA, no significant correlation was identified between CD163 expression on IHC and the studied SNPs in CHL patients ( $\mathrm{P}=0.690)$, although the rs75608120 polymorphism may represent a separate single genetic predictive biomarker for CHL prognosis. In addition, the lack of an association between CD163 IHC and its studied SNPs in CHL may be due to the small number of CHL patients included in the present study. Further investigation is required to study more or even all the SNPs at the promoter region; however, this may indicate that the protein overexpression of CD163 on TAMs in CHL cases is not directly caused by any genetic polymorphism in its gene, but rather the result of immunological pathophysiology of TME crosstalk.

In conclusion, the present study provided valuable insight into the molecular genetics and antigenicity of CD163 and its effect on the prognosis of CHL in Saudi patients. CD163 overexpression on TAMs was confirmed to be significantly associated with early relapse and reduced survival post-therapy. To the best of our knowledge, the present study was also the 
first to define a specific genetic pattern that is clearly associated with the clinical outcome of CHL. Accordingly, CD163 is a useful predictive antigenic and genetic biomarker for CHL prognosis in Saudi patients. Our findings may pave the way for improving the clinical management of CHL in the future through molecular analyses for gene expression and IHC, and they may help develop better therapeutic protocols incorporating pharmacogenetics and monoclonal antibodies specifically designed to block TAM receptors for CD163. Anti-CD163 antibodies may be applied for the treatment of HL along with the currently available therapeutic options.

\section{Acknowledgements}

Warmest thanks to my colleagues at Arabian Gulf University, Mr. Ali Mehaimeed, Mr. Muhallab, Dr Nouriddin and Dr Sonia Bourguiba, as well as Mr. AbuAqla, Mr. Khalid Khunaizi and Dr Ali Rabaan at Johns Hopkins Aramco Healthcare (JHAH), who provided the necessary technical assistance and efficient support. Special thanks to Dr Ahmed Alsagheir and Dr Adel Alkhatti from the Oncology institute at JHAH. I am grateful to have such support from all the pioneers and philanthropists around me.

\section{Funding}

The present study was supported by the Arabian Gulf University-College of Medicine and Medical Sciences, Bahrain. The funder had no role in the study design, data collection and analysis, decision to publish, or preparation of the manuscript.

\section{Availability of data and materials}

The datasets generated and analyzed during the present study are available from the corresponding author on reasonable request.

\section{Authors' contributions}

HSA and WR designed the experiments; HSA performed the experiments, data validation and formal analysis, and wrote the manuscript; WR, AD and MF reviewed and revised the manuscript; AD acquired funding. All authors have read and approved the final version of this manuscript.

\section{Ethics approval and consent to participate}

Ethics approval for the present study was obtained from the Institutional Review Board. Written informed consent was provided by the subjects regarding the use of their tissue samples for research purposes.

\section{Patient consent for publication}

Not applicable.

\section{Competing interests}

The authors declare that they have no competing interests.

\section{References}

1. Rauf MS, Akhtar S and Maghfoor I: Changing trends of adult lymphoma in the kingdom of Saudi Arabia-comparison of data sources. Asian Pac J Cancer Prev 16: 2069-2072, 2015.

2. Leukemia and Lymphoma Society: American Cancer Society Cancer Facts and Figures 2016; GLOBOCAN 2012; ClinicalTrials. gov; CRI grantee progress reports and other CRI grantee documents. 2016. http://www.cancerresearch.org/cancer-immunotherapy/impacting-all-cancers/lymphoma\#sthash.9EkuimDH. dpuf 91. Accessed November 14, 2014.

3. Al-Madouj AN, Eldali A and Al-Zahrani AS: Ten year cancer incidence among nationals of the GCC states. Gulf center for cancer control and prevention, king faisal specialist hospital and research center. 2011. http://www.moh.gov. bh/Content/Files/Publications/GCC\%20Cancer\%20Incidence\%20 2011.pdf. Accessed September 1, 2011.

4. Cancer Incidence Report Saudi Arabia 2013. http://www.chs.gov. $\mathrm{sa} / \mathrm{Ar} / \mathrm{HealthCenters/NCC/CancerRegistry/CancerRegistryReports/}$ 2013. Accessed June 1, 2016.

5. Maggioncalda A, Malik N, Shenoy P, Smith M, Sinha R and Flowers CR: Clinical, molecular, and environmental risk factors for Hodgkin lymphoma. Adv Hematol 2011: 736261, 2011.

6. Al-Diab AI, Siddiqui N, Sogiawalla FF and Fawzy EM: The changing trends of adult Hodgkin's disease in Saudi Arabia. Saudi Med J 24: 617-622, 2003.

7. Piccaluga PP, Agostinelli C, Gazzola A, Tripodo C, Bacci F, Sabattini E, Sista MT, Mannu C, Sapienza MR, Rossi M, et al: Pathobiology of hodgkin lymphoma. Adv Hematol 2011: 920898, 2011.

8. Montes-Moreno S: Hodgkin's lymphomas: A tumor recognized by its microenvironment. Adv Hematol 2011: 142395, 2011.

9. Smith LB: Nodular lymphocyte predominant Hodgkin lymphoma: Diagnostic pearls and pitfalls. Arch Pathol Lab Med 134: 1434-1439, 2010.

10. Carbone PP, Kaplan HS, Musshoff K, Smithers DW and Tubiana M: Report of the committee on Hodgkin's disease staging classification. Cancer Res 31: 1860-1861, 1971.

11. Hasenclever D and Diehl V: A prognostic score for advanced Hodgkin's disease. International prognostic factors project on advanced Hodgkin's disease. N Engl J Med 339: 1506-1514, 1998.

12. Canioni D, Deau-Fischer B, Taupin P, Ribrag V, Delarue R, Bosq J, Rubio MT, Roux D, Vasiliu V, Varet B, et al: Prognostic significance of new immunohistochemical markers in refractory classical Hodgkin lymphoma: A study of 59 cases. PLoS One 4: e6341, 2009.

13. Moccia AA, Donaldson J, Chhanabhai M, Hoskins PJ, Klasa RJ, Savage KJ, Shenkier TN, Slack GW, Skinnider B, Gascoyne RD, et al: International prognostic score in advanced-stage Hodgkin's lymphoma: Altered utility in the modern era. J Clin Oncol 30: 3383-3388, 2012.

14. Henry-Amar M, Friedman S, Hayat M, Somers R, Meerwaldt JH, Carde P, Burgers JM, Thomas J, Monconduit M and Noordijk EM: Erythrocyte sedimentation rate predicts early relapse and survival in early-stage Hodgkin disease. The EORTC lymphoma cooperative group. Ann Intern Med 114: 361-365, 1991.

15. Roshal M, Wood BL and Fromm JR: Flow cytometric detection of the classical hodgkin lymphoma: Clinical and research applications. Adv Hematol 2011: 387034, 2011.

16. Dunphy CH: Applications of flow cytometry and immunohistochemistry to diagnostic hematopathology. Arch Pathol Lab Med 128: 1004-1022, 2004.

17. Scott DW and Steidl C: The classical Hodgkin lymphoma tumor microenvironment: Macrophages and gene expression-based modeling. Hematology Am Soc Hematol Educ Program 2014: 144-150, 2014.

18. Tan KL, Scott DW, Hong F, Kahl BS, Fisher RI, Bartlett NL, Advani RH, Buckstein R, Rimsza LM, Connors JM, et al: Tumor-associated macrophages predict inferior outcomes in classic Hodgkin lymphoma: A correlative study from the E2496 Intergroup trial. Blood 120: 3280-3287, 2012.

19. Mallmann MR, Schmidt SV and Schultze JL: Macrophages in human cancer: Current and future aspects. Atlas Genet Cytogenet Oncol Haematol 16: 765-774, 2012.

20. Ruhrberg C and De Palma M: A double agent in cancer: Deciphering macrophage roles in human tumors. Nat Med 16: 861-862, 2010. 
21. Ree HJ and Kadin ME: Macrophage-histiocytes in Hodgkin's disease. The relation of peanut-agglutinin-binding macrophage-histiocytes to clinicopathologic presentation and course of disease. Cancer 56: 333-338, 1985.

22. Guo B, Cen H, Tan X and Ke Q: Meta-analysis of the prognostic and clinical value of tumor-associated macrophages in adult classical Hodgkin lymphoma. BMC Med 14: 159, 2016.

23. Sud A, Thomsen H, Sundquist K, Houlston RS and Hemminki K: Risk of second cancer in Hodgkin lymphoma survivors and influence of family history. J Clin Oncol 35: 1584-1590, 2017.

24. Steidl C, Lee T, Shah SP, Farinha P, Han G, Nayar T, Delaney A Jones SJ, Iqbal J, Weisenburger DD, et al: Tumor-associated macrophages and survival in classic Hodgkin's lymphoma. N Engl J Med 362: 875-885, 2010

25. Sánchez-Espiridión B, Montalbán C, López A, Menárguez J, Sabín P Ruiz-Marcellán C, Lopez A, Ramos R, Rodríguez J, Cánovas A, et al: A molecular risk score based on 4 functional pathways for advanced classical Hodgkin lymphoma. Blood 116: e12-17, 2010.

26. Gregory AD and Houghton AM: Tumor-associated neutrophils: New targets for cancer therapy. Cancer Res 71: 2411-2416, 2011.

27. Wahl LM and Kleinman HK: Tumor-associated macrophages as target for cancer therapy. J Natl Cncer Inst 90: 1583-1584, 1998.

28. Derenzini E and Younes A: Predicting treatment outcome in classical Hodgkin lymphoma: Genomic advances. Genome Med 3: 26, 2011

29. Barros MH, Segges P, Vera-Lozada G,Hassan R and Niedobitek G: Macrophage polarization reflects $\mathrm{T}$ cell composition of tumor microenvironment in pediatric classical Hodgkin lymphoma and has impact on survival. PLoS One 10: e0124531, 2015.
30. Yoon DH, Koh YW, Kang HJ, Kim S, Park CS, Lee SW, Suh C and Huh J: CD68 and CD163 as prognostic factors for Korean patients with Hodgkin lymphoma. Eur J Haematol 88: 292-305, 2012.

31. Azambuja D, Natkunam Y, Biasoli I, Lossos IS, Anderson MW, Morais JC and Spector N: Lack of association of tumor-associated macrophages with clinical outcome in patients with classical Hodgkin's lymphoma. Ann Oncol 23: 736-742, 2012.

32. Lau SK, Chu PG and Weiss LM: CD163: A specific marker of macrophages in paraffin-embedded tissue samples. Am J Clin Pathol 122: 794-801, 2004.

33. Ritter M, Buechler C, Langmann T, Orso E, Klucken J and Schmitz G: The scavenger receptor CD163: Regulation, promoter structure and genomic organization. Pathobiology 67: 257-261, 1999.

34. Harris JA, Jain S, Ren Q, Zarineh A, Liu C and Ibrahim S: CD163 versus CD68 in tumor associated macrophages of classical Hodgkin lymphoma. Diagn Pathol 7: 12, 2012.

35. El-Zein R, Monroy CM, Etzel CJ, Cortes AC, Xing Y, Collier AL and Strom SS: Genetic polymorphisms in DNA repair genes as modulators of Hodgkin disease risk. Cancer 115: 1651-1659, 2009.

This work is licensed under a Creative Commons Attribution-NonCommercial-NoDerivatives 4.0 International (CC BY-NC-ND 4.0) License. 